\title{
EFECTO DE LA QUIMIOTERAPIA SOBRE LA MICROBIOTA SUBGINGIVAL Y RECUENTO DE LEVADURAS EN SALIVA EN PACIENTES DE 6-12 AÑOS CON LEUCEMIA LINFOCÍTICA AGUDA (L.L.A) DEL HOSPITAL UNIVERSITARIO DEL VALLE
}

\author{
Katya Botero Torres \\ Javier Enrique Botero Torres 2 \\ Martha Lucia Tello Garcia \\ Adriana Jaramillo ${ }^{2}$
}

Palabras claves: Leucemia Linfocítica Aguda, quimioterapia, microbiota subgingival, levaduras

\section{RESUMEN}

La leucemia linfocitica aguda (L.L.A) se puede presentar a cualquier edad pero es más común en niños. Las complicaciones orales más comunes después de la quimioterapia son la mucositis, infecciones locales, disfunción de las glándulas salivales, disfunción en el gusto, dolor y hemorragias. El propósito de la presente investigación fue determinar los efectos de la quimioterapia sobre la microbiota subgingival y el recuento de levaduras en saliva en pacientes de 6 a 12 años con L.L.A en el Hospital Universitario del Valle. Se estudiaron 12 niños ( 8 hombres $/ 4$ mujeres) con diagnóstico de L.L.A que inició su primer ciclo de quimioterapia y/o pacientes que regresaban de un periodo de descanso mayor de 3 meses de Enero a Julio del 2002. A los 12 pacientes se les tomó una primera muestra subgingival y una muestra de saliva no estimulada, una segunda muestra a $\operatorname{los} 30$ dias después de haber recibido su primer cicio de quimioterapia y una tercera muestra a los dos meses. El análisis estadístico incluyo el Chi cuadrado de Pearson, test de Friedman y Test de Cochran $(\mathrm{P}=0.05$ ) empleando el software SSPS (ver 10.0) para Windows.
La mucositis y la gingivitis fueron las complicaciones orales más frecuentes. El Fusobacterium spp. y los bacilos entéricos gram negativos fueron los microorganismos más prevalentes de la microbiota subgingival favorecida probablemente por la rata de secreción salival disminuida y una reducción en el pH intraoral causados por la quimioterapia. A medida que el flujo salival disminuye existe una tendencia a aumentar el recuento de levaduras en saliva lo cual podría general una mayor susceptibilidad al desarrollo de candidiasis.

\section{INTRODUCCION}

La leucemia es una enfermedad hematológica progresiva malignizante que está caracterizada por una proliferación y desarrollo anormal de los leucocitos y sus precursores en sangre y médula ósea ${ }^{1}$. La etiología de la enfermedad es desconocida pero se sospechan causas como infecciones virales, exposición a radiación ionizante, radiación electromagnética, exposición química, alteraciones citogenéticas y daños cromosómicos ${ }^{2}$. La leucemia linfocitica aguda (L.L.A.) se puede presentar a cualquier edad pero es más común en niños y es frecuentemente tratada con quimioterapia ${ }^{2}$. Las complicaciones orales más comunes observadas después de las terapias contra el cáncer son la mucositis, las infecciones locales, la disfunción de las glándulas salivares, la disfunción en el gusto, el

Universidad del Valle, Grupo de Medicina Periodontal.

Odontóloga especialista en Odontopediatria y Ortopedia maxilar. Universidad del Valle. 
dolory las hemorragias ${ }^{3}$. Una vez que se vea afectada la integridad mucosa, infecciones sistémicas y locales pueden ser causadas por la flora oral nativa, así como también por organismos nosocomiales y oportunistas ${ }^{4,5}$. La Candida se puede encontrar como un miembro más de la microbiota oral en un $30-40 \%$ de bocas clínicamente sanas, pero bajo ciertas condiciones desfavorables, como la hipofunción de las glándulas salivales ${ }^{6.78}$ que llevan a la alteración de la microbiota oral, puede causar formas severas de candidiasis oral $^{4,9,10,11}$. Existen indicios muy sólidos que indican que los medicamentos utilizados para la terapia del cáncer y la radiación también aumentan la virulencia de las células de Candida, además que se ha observado un incremento en la adherencia de la Candida a la mucosa oral durante y después del tratamiento ${ }^{12}$. Son muy pocos los estudios realizados sobre los cambios en la microbiota oral durante los diferentes periodos de tratamiento y la mayoría de estos son hechos con adultos. Es necesario el conocimiento de los cambios de la microbiota subgingival ocasionados con la quimioterapia ya que pueden ser determinantes en el desarrollo de enfermedades periodontales en niños leucémicos. El propósito de la presente investigación fue determinar los efectos de la quimioterapia sobre la microbiota subgingival y el recuento de levaduras en saliva en pacientes de 6 a 12 años con leucemia linfocítica aguda (L.L.A) en el Hospital Universitario del Valle.

\section{MATERIALES Y METODOS}

Este es un estudio descriptivo clínico-microbiológico en el cual se realizó la identificación de la microbiota subgingival, recuento de levaduras en saliva y hallazgos clínicos de niños con L.L.A observando los cambios que se presentaron en ellos durante dos meses de seguimiento. La selección de los pacientes se hizo por conveniencia. El estudio fue previamente aprobado por el comité de ética en humanos de la Facultad de Salud de la Universidad del Valle y como se trataba de menores de edad, los padres firmaron un consentimiento informado escrito. Los Criterios de inclusión fueron: pacientes con L.L.A los cuales iniciaron su primer ciclo de quimioterapia y/o pacientes que regresaban después de un periodo de descanso de quimioterapia mayor de 3 meses en el H.U.V (Hospital Universitario del Valle) que participaron voluntariamente en el estudio, pacientes en dentición mixta de $6-12$ años, pacientes que únicamente recibieron quimioterapia para el manejo de L.L.A, pacientes sin caries y con un valor mínimo de recuento de plaquetas aceptable para la realización del procedimiento de 100.000 $\mathrm{pq} / \mathrm{ml}$ y pacientes con consentimiento informado. Los criterios de exclusión fueron: pacientes que presentaron otra enfermedad sistémica además de la L.L.A, pacientes que recibian medicamentos diferentes al protocolo de quimioterapia o que recibieron tratamiento con antibióticos hasta un mes antes de la toma de la muestra.

Se tomaron 12 niños ( 8 hombres y 4 mujeres) con diagnóstico de L.L.A que iniciaron su primer ciclo de quimioterapia y/o pacientes que regresaban de un período de descanso mayor de 3 meses del Hospital Universitario del Valle de Enero a Julio del 2002. A los 12 pacientes se les realizó un exámen clínico intraoral, una muestra microbiológica subgingival y una muestra de saliva no estimulada en tres periodos; el primer periodo ( $\sin$ haber recibido la primera dosis de quimioterapia), en el segundo periodo (a los 30 días después de haber recibido su primer ciclo de quimioterapia) y en el tercer periodo (a los dos meses de haber recibido su primer ciclo de quimioterapia). ( protocolo de quimioterapia de alto riesgo utilizado en el Hospital Universitario del Valle).Se obtuvieron 36 muestras de saliva y 36 muestras microbiológicas subgingivales. Todos los niños fueron ambulatorios en el servicio de quimioterapia durante el periodo del estudio.

Medidas clínicas

En el tiempo transcurrido entre Enero de 2002 y Julio de 2002, se tomaron registros de signos cli- 
nicos de lesiones orales (mucositis, candidiasis, úlceras, herpes virus, aftas, queilitis angular, absceso de origen dentario, pericoronitis, leucoplasias, aumento tisular por trauma), indices gingivales según Löe y Silness (1963) $)^{13} \mathrm{e}$ indices periodontales $\left(\right.$ ICPNT $\left.^{14}\right)$.

\section{MUESTRA MICROBIOLÓGICA}

Antes de tomar la muestra microbiológica subgingival y el sondaje periodontal se revisó el cuadro hemático con recuento de plaquetas que se le realiza a cada paciente como requisito para aplicar la dosis de quimioterapia. Se tomaron 3 muestras subgingivales por cada paciente en $\mathrm{el}$ primer molar inferior derecho por vestibular; se aisló con rollos de algodón, se eliminó la placa supragingival y se insertó una punta de papel estéril $^{15}$ hasta el fondo del surco durante 15 segundos. Las puntas de papel fueron colocadas en un frasco estéril con medio de transporte VMGAIII. Las muestras fueron procesadas en el laboratorio de microbiologia oral de la Universidad del Valle, máximo a las 24 horas siguientes a la toma de la muestra, aunque el medio VMGA III es capaz de mantener la viabilidad de organismos anaerobios orales hasta por tres dias. Las muestras fueron procesadas a temperatura ambiente e inmediatamente incubadas en los sistemas de cultivo anaerobio (Aaneropack System $\mathbb{B}$ : $\mathrm{CO}_{2}$ Oxoid System $(\mathcal{B})$. Para la detección de anaeróbicos facultativos se utilizo un medio selectivo TSBV (tripticasa - soya - agar suplementado con $75 \mathrm{ug} /$ $\mathrm{ml}$ de Bacitracina $-5 \mathrm{mg} / \mathrm{ml}$ de Vancomicina y $5 \%$ de suero estéril de caballo). Se determinaron los porcentajes bacterianos en cada placa de agar y se utilizaron los métodos de identificación de Slots (Slots 1.986) para identificación bacteriana presuntiva. Luego fueron incubados por 3 días en un ambiente con $5 \%$ de $\mathrm{CO}_{2}$.

\section{MUESTRA DE SALIVA:}

Las muestras de saliva fueron recolectadas bajo condiciones estandarizadas. Se tomaron entre las
8:00 y 9:30 am, hora en que los pacientes asistian al servicio de consulta externa de quimioterapia del H.U.V. Los pacientes no habían realizado medidas de higiene oral ni habían consumido alimentos una hora antes de la toma de la muestra. A los niños se les enseñó como escupir la saliva acumulada en un tubo plástico estéril durante tres minutos. Las muestras fueron transportadas en un medio refrigerado y llevadas al Laboratorio de Micología de la Universidad del Valle dentro de la media hora siguiente. Al llegar la muestra de saliva al laboratorio se realizó la medición de la cantidad de saliva con micropipeta desechando la parte espumosa. El flujo de saliva normal sin estimular para este grupo de niños se definió basados en los hallazgos del estudio realizado por Brezzetal ${ }^{16}$, tomando como normal 0.61 mililitros /min y valores por debajo de éste se consideraron como hiposalivación. La rata de secreción salival fue calculada en mililitros/minuto. Luego se sembró en cajas de petri con agar saboraud y agar micosel realizando la extensión con un asa de vidrio. Se incubaron a $37 \mathrm{oC}$ durante 48 horas y se realizó el conteo de las levaduras las cuales se expresan en Unidades Formadoras de Colonias por mililitro (UFC/ml). Para realizar la correlación entre signos y sintomas de candidiasis tomamos como valor de referencia un recuento en saliva de Candida mayor de 400 UFC $^{17,18}$.

\section{ANALISIS ESTADISTICO}

Para el análisis descriptivo univariado y bivariado se empleó sofware SPSS versión 10.0 para Windows. Para establecer diferencias o similitudes entre las diferentes variables estudiadas en los tres periodos de observación se utilizó el test de Friedman. Para establecer la correlación entre cada microorganismo con el índice gingival y periodontal de cada paciente, se utilizó el Chi cuadrado de Pearson. Con relación a la frecuencia de aparición de cada microorganismo se utilizó el Test de Cochran. Una probabilidad inferior o igual a 0.05 ( $\mathrm{P}=0.05)$ se consideró como significativa. Para establecer la correlaciỏn entre la rata de 
secreción salival con el recuento de levaduras en agar Saboraud y el recuento de levaduras en agar Micosel en cada uno de los periodos de observación, se utilizó el coeficiente de correlación de Pearson ( $\mathrm{P}=0.05$ ) con $95 \%$ de confianza.

\section{RESULTADOS}

En el presente estudio se determinó la microbiota subgingival y recuento de levaduras en saliva en 12 niños, ( 8 hombres y 4 mujeres) con diagnóstico de L.L.A de los cuales 2 iniciaron su primer ciclo de quimioterapia y 10 pacientes regresaban de un período de descanso mayor de 3 meses. La edad promedio fue 8.3 años ( rango $6-12$ años ) que recibieron tratamiento ambulatorio en el Hospital Universitario del Valle (Tabla 1). Se

Tabla 1. Descripción demográfica de la muestra.

\begin{tabular}{|c|c|c|c|c|}
\hline & \\
\hline & & $n$ & porcentaje & total \\
\hline \multirow{3}{*}{ Género } & Masculino & 8 & 66.7 & \multirow{3}{*}{$12(100 \%)$} \\
\hline & & & & \\
\hline & Femenino & 4 & 33.3 & \\
\hline \multirow{3}{*}{ Edad } & Minima & 6 & & \\
\hline & máxima & 12 & & \\
\hline & promedio & 8.33 & & \\
\hline \multirow{3}{*}{ Ciclo de quimioterapia } & Nuevo & 2 & 16.7 & \multirow{3}{*}{$12(100 \%)$} \\
\hline & & & & \\
\hline & Descanso & 10 & 83.3 & \\
\hline
\end{tabular}

obtuvieron un total de 36 muestras de saliva y 36 muestras subgingivales. Al hacer el examen clinico en el primer periodo no se encontró evidencia de lesiones orales en los pacientes. En el segundo periodo y tercer periodo se encontraron 8 y 4 casos respectivamente con lesiones orales siendo las más frecuentes gingivitis y mucositis (Tabla 2). Al realizar el examen clinico durante los tres periodos respecto al indice gingival y periodontal,

Tabla 2. Lesiones orales encontradas durante los 3 periodos de observación.

\begin{tabular}{|l|c|c|c|}
\hline \multicolumn{1}{|c|}{ Lesion } & primer periodo & scgundo periodo & tercer periodo \\
\hline Mucositis & 0 & $5(41.7 \%)$ & $2(16.7 \%)$ \\
\hline Queilitis anquiar & 0 & $3(25 \%)$ & 0 \\
\hline Ulcera traumatica & 0 & 0 & $1(8.3 \%)$ \\
\hline herpes simple periotal & 0 & 0 & $1(8.3 \%)$ \\
\hline Gingivitis & $5(41.7 \%)$ & $12(100 \%)$ & $11(91.7 \%)$ \\
\hline
\end{tabular}

en el primer periodo se observó una alta frecuencia de IG 0 ( 7 casos $58.3 \%$ ) e IP 0 ( 8 casos $66.7 \%$ ), en el segundo periodo IG2 e IP1 (10 casos $83.3 \%$ ) y en el tercer periodo IG2 ( 6 casos $50 \%$ ) e IP 0 ( 8 casos $66.7 \%$ ). (Tabla 3 ).

De los 12 casos estudiados el tipo de microbiota periodontopática predominante en el primer periodo fueron los bacilos entéricos gram (-) (6 casos $50 \%$ ), Fusobacterium spp. y Eubacterium spp. ( 5 casos $41,7 \%$ ) (Tabla 4). En dos de los pacientes se logró la identificación específica para bacilos entéricos gram (-) encontrando Kleisella pneumoniae y Pseudomonas aeruginosa. Los porcentajes de la microbiota subgingival de los bacilos entéricos gram (-) en 4 de los casos positivos representaron el $100 \%$ de la microbiota cultivada. Para el segundo periodo los más predominantes fueron el Fusobacterium spp. (10 casos $83.7 \%)$, bacilos entéricos gram (-), Porphyromona gingivalis ( 6 casos $50 \%$ ) y Eikenella corrodens (5 casos $41.7 \%$ ) (Tabla 4). Los porcentajes de la microbiota subgingival de los bacilos entéricos gram (-) en 2 de los casos positivos durante éste segundo período, representaron el $100 \%$ de la microbiota cultivada. Para el tercer periodo los más predominantes fueron el Fusobacterium spp. (12 casos 100\%), Campylobacter spp. (7 casos 58.3\%), Eubacterium spp. (6 casos $50 \%$ ) (Tabla 4). $\mathrm{Al}$ realizar la correlación entre la microbiota subgingival cultivada con el indice gingival y periodontal no se encontró estadísticamente significativa.

Análisis de saliva:

En los 12 casos estudiados se encontró en el primer periodo un promedio de rata de secreción salival de $0.85 \mathrm{ml} /$ minuto, luego disminuyó a 0.44 $\mathrm{ml} /$ minuto en el segundo periodo (Friedman test) $y$ en el tercer periodo observamos un leve aumento a $0,48 \mathrm{ml} /$ minuto $(p=0.001)$. La correlación de la rata de secreción salival en los tres periodos fue éstadisticamente significativa (friedman test) (Gráfico 1). 
TABLA 4. Número de casos, porcentaje,porcentaje promedio microbiota subgingival cultivada, rango,promedio total de colonias

\begin{tabular}{|c|c|c|c|c|c|c|c|c|c|c|c|c|c|c|c|}
\hline & \multicolumn{5}{|c|}{ PAIMER PEAIODO } & \multicolumn{5}{|c|}{ SEGUNDO PERIODO } & \multicolumn{5}{|c|}{ TERCER PEFIODO } \\
\hline & $n$ & porcentajel & $\%$ Promedio micro.sub. & rango & promedio T.C & $n$ & porcentajel & \% Promedio micro.sub. & rango & promedio T.C. & $n$ & porcentaje & \% Promedio micro.sub. & |rango| & promedio T.C \\
\hline A.a & 0 & 0 & 0 & 0 & 0 & 1 & 8.3 & $8.33 \times 10^{2}$ & $0-1$ & 0.17 & 2 & 16.7 & 0.25 & $0-2$ & 0.43 \\
\hline$P . g$ & 3 & 25 & 0.5 & $0-2$ & 0.63 & 6 & 50 & 1.0975 & $0-5$ & 1.83 & 4 & 33.3 & 0.64 & $0-4$ & 0.73 \\
\hline P.i & 3 & 25 & 0.63 & $0-3$ & 1.29 & 4 & 33.7 & 0.86 & $0-8$ & 1.08 & 2 & 16.7 & 0.13 & $0-1$ & 0.17 \\
\hline B.t & 0 & 0 & 0 & 0 & 0 & 1 & 8.3 & $4.17 \times 10^{-2}$ & $0-1$ & $8.33 \times 10^{2}$ & 0 & 0 & 0 & 0 & 0 \\
\hline C.s & 3 & 25 & 0.14 & $0-1$ & 0.37 & 1 & 8.3 & 0.11 & 0.1 & 0.23 & 7 & 58.3 & 0.83 & $0-3$ & 1.04 \\
\hline E.s & 5 & 41.7 & 1.18 & 0.10 & 0.96 & 4 & 33.3 & 0.32 & $0-1$ & 0.64 & 6 & 50 & 1.21 & 0.4 & 1.62 \\
\hline$F, s$ & 5 & 41.7 & 1.35 & 0.7 & 2 & 10 & 83.7 & 5.65 & $0-22$ & 10.33 & 12 & 100 & 6.87 & $1-20$ & 10.88 \\
\hline$P . m$ & 1 & 8.3 & 0.52 & 0.3 & 0.54 & 2 & 16.7 & 0.60 & 0.6 & 0.75 & 4 & 33.3 & 0.64 & 0.5 & 1.54 \\
\hline E.c & 0 & 0 & 0 & 0 & 0 & 5 & 41.7 & 0.64 & $0-3$ & 0.82 & 2 & 16.7 & 8.42 & $0-100$ & 1.75 \\
\hline$D, \rho$ & 1 & 8.3 & $8.33 \times 10^{2}$ & 0.1 & 2 & 0 & 0 & 0 & 0 & 0 & 4 & 33.3 & 0.46 & 0.3 & 0.73 \\
\hline Bac. Ent & 6 & 50 & 35.85 & $0-100$ & 83.79 & 6 & 50 & 33.93 & $0-100$ & 73.71 & 2 & 16.7 & 6.71 & 0.80 & 13.42 \\
\hline
\end{tabular}

$n:$ Número de casos

$\%$ promedio micro sub. porcentaje promedio microbiota subgingival

promedio T.C: Promedio tamaño de colonias.

A:a : Actinobacillus actinomycetemcomitans

P.g: Porphyromonas gingivalis

P.1: Prevotella intermedia

B.t: Bacteroides forsythus

C.s: Campylobacter spp

E.s : Eubacterium spp

F.s: Fusabacterium spo

P.m: Peptostroptococcus micros

E.c: Elkenella corrodens

D.p: Dialister pneumosintes

Bact Ent.: bacilos entericos gram negativos 
Tabla 5. Recuento de levaduras en agar Saboraud segùn periodo

\begin{tabular}{|l|c|c|c|c|c}
\hline & primer periodo segundo periodo tercer periodo test friedman test cochran \\
\hline Casos positivos & $7(58.3 \%)$ & $8(58.3 \%)$ & $5(41.7 \%)$ & 0.328 & 0.311 \\
\hline rango & $0-1104$ & $0-1532$ & $0-1812$ & & \\
\hline promedio U.F.C/ ml. & 129.83 & 173.67 & 160 & & \\
\hline p & & 0.848 & 0.756 & & \\
\hline
\end{tabular}

$p$ significante $p \leq 0.05$

Tabla 6. Recuento de levaduras en agar Micosel según periodo.

\begin{tabular}{|l|c|c|c|c|c|}
\hline & primer periodo & segundo periodo & tercer periodo & test friedman & test cochran \\
\hline Casos positivos & $6(50 \%)$ & $8(33.3 \%)$ & $3(25 \%)$ & & \multirow{2}{*}{0.311} \\
\cline { 1 - 4 } rango & $0-900$ & 0.1544 & 0.1700 & 0.368 \\
\cline { 1 - 4 } promedio U.F.C/ ml. & 94.33 & 151.33 & 147 & & \\
\cline { 1 - 4 } correlación pearson & & 0.846 & 0.757 & & \\
\hline
\end{tabular}

p significante $p \leq 0.05$

que una de las infecciones virales más comunes en pacientes que reciben quimioterapia es el virus del Herpes Simplex tipo 1, en este estudio solo se encontró un caso en el tercer periodo debido probablemente a que estos niños por su corta edad aún no han tenido contacto con el virus del herpes simplex tipo $1^{23} 24$. Durante el segundo periodo todos los pacientes presentaron signos clínicos de gingivitis. Peterson ${ }^{25}$ demostró que las infecciones gingivales son relativamente comunes en pacientes que reciben terapia mielosupresiva. La aparición de esta condición puede deberse a que en pacientes que reciben quimioterapia, la microbiota oral cambia a una predominantemente gram negativa ${ }^{25}$. También se encontró que uno de los microorganismos más prevalentes en la microbiota subgingival de los 12 pacientes con L.L.A (612 años) son los bacilos entéricos gram negativos. Diferentes estudios han observado la prevalencia de bacilos gram negativos y levaduras en pacientes con leucemia antes de iniciar el tratamiento de quimioterapia, encontrando una alta prevalencia de bacilos entéricos gram negativos, Pseudomonas y levaduras ${ }^{26.27} .28$. La presencia de los bacilos entéricos se ha asociado con hospitalización, terapia antibiótica y rata de secreción salival disminuida concomitantemente con una reducción del pH intraoral que puede favorecer su adherencia a las células del epitelio bucal ${ }^{29}$. Dentro de los periodontopáticos predominantes en este estudio se encontraron el Fusobacterium spp. P.gingivalis, Eubacterium, E. corrodens. Moore y $\mathrm{col}^{30}$ reportaron un número incrementado de Fusobacterium spp. en pacientes con gingivitis, siendo el Fusobacterium spp. la causa principal de esta condición. Similar a lo resultados de Moore ${ }^{31}$ sobre la prevalencia de Fusobacterium spp. en niños, en este estudio se encontró un alto porcentaje de este microorganismo antes de recibir la quimioterapia. Es raro encontrar $P$. gingivalis en el ambiente subgingival de niños prepuberales debido a que los factores nutritivos y ambientales no son los adecuados para su crecimiento ${ }^{32}$ y cuando aparece, es un microorganismo dificil de cultivar e identificar ${ }^{33}$, sin embargo en este estudio se presentó en $6 \mathrm{ca}$ $\operatorname{sos}(50 \%)$ en el segundo periodo. Se ha visto que la prevalencia de $P$. gingivalis y Bacteroides. 


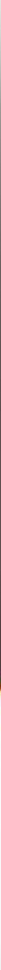


forsytus está entre $14 \%$ y $8 \%$ respectivamente detectados mediante la reacción en cadena de la polimerasa en niños entre $\operatorname{los} 2$ y 11 años ${ }^{34}$, estos dos microorganismos aumentan su prevalencia y número cuando se incrementa la profundidad de la bolsa ${ }^{35}$. Sólo se encontró 1 caso de $B$. forsythus. Moore et $\mathrm{al}^{36}$ han reportados niveles aumentados de Eubacterium en sitios periodontalmente afectados, principalmente de Eubacterium nodatum y Eubacterium D11, sin embargo otros miembros de esta especie se encuentran en niveles altos tanto en salud como en enfermedad. Se encontró la presencia de Eubacterium spp en porcentajes similares en los tres periodos $(41.7 \%)$ sin observar un cambio significativo entre ellos. La E corrodens se detecta frecuentemente en la microbiota subgingival. Goldenstein ${ }^{37}$ indicó que este microorganismo se presenta en el $90 \%$ de los niños entre los 7 y 14 años de edad pero solo un $79 \%$ en los adultos. Ashimoto et al. ${ }^{38}$ encontraron una prevalencia de $E$. corrodens de $66 \%$ en niños entre los 2 y 11 años de edad. La mayor prevalencia de $E$. corrodens se encontró en el segundo periodo con un total de 5 casos $(41.7 \%)$. En el presente estudio se observó una disminución significativa en la rata de secreción salival entre los tres periodos. Se ha reportado en varios estudios una disminución en la rata de secreción salival en pacientes que reciben quimioterapia $8,39,40,41,42$ aunque algunos investigadores no han encontrado diferencias significativas ${ }^{43,44}$. Se sabe que la leucemia aguda no influye directamente sobre la rata de secreción salival, sin embargo, al inicio de toda terapia citotóxica los pacientes están muy ansiosos $\mathrm{s}^{45}, \mathrm{y}$ además el estrés emocional que presentan hace que se reduzca la rata de secreción salival ${ }^{46,47}$. En el primer periodo se encontró un promedio de rata de secreción salival de 0.85 mililitros/minuto, similar a la reportada en otros estudios en niños que no reciben medicación ${ }^{16}$. Wahlin ${ }^{39}$ en 1991 en su estudio encontró que al inicio del tratamiento la rata de secreción salival fue más baja que en personas saludables y entre los muchos factores que él encontró como posible causa, está la ansiedad que estos pacientes presentan al inicio del primer ciclo de quimioterapia. Se observó hiposalivación en el segundo periodo con una rata de secreción salival promedio de 0.44 mililitros / minuto (Friedman test). Otros factores que también contribuyen a ésta disminución son el daño indirecto a las glándulas salivales por las drogas citotóxicas y la deshidratación producida por una disminución en la ingesta de alimentos y líquidos debido a los efectos colaterales que produce la quimioterapia ${ }^{39}$. Debido a los muchos factores involucrados en la disminución de la rata de secreción salival (tipos de drogas citotóxicas, deshidratación, ansiedad, y métodos de administración y dosis), los resultados de este estudio deben ser interpretados con precaución debido al tamaño del grupo de estudio y a que en su diseño no estaba incluida la evaluación y control de estas variables externas. Otro aspecto negativo que presentan los pacientes con cáncer que son sometidos a quimioterapia es el incremento en la aparición de infecciones fúngicas especialmente Candidia$\operatorname{sis}^{6,48,49}$. La hipofunción de las glándulas salivales puede alterar la microbiota oral e incrementar el riesgo de infección ${ }^{7,50,51,52}$. En este estudio la frecuencia de colonización por Candida en el primer y segundo periodo fue $58.3 \%$ y en el tercer periodo $41.7 \%$, similar a la observada en otros estudios $(45-70 \%)^{6,17}$. Se ha encontrado una relación inversa entre la rata de secreción salival y el conteo de Candida en saliva ${ }^{33}$. Al realizar la correlación entre la cantidad de saliva y el recuento de levaduras tanto en agar micosel como en agar sabouraud, se encontró que a medida que el flujo salival disminuye existe una tendencia a aumentar el recuento de levaduras en saliva, siendo esto mayor en el segundo periodo sin ser estadisticamente significativa. Diferentes autores han reportado que no han encontrado correlación entre la evidencia clínica y el recuento de levaduras al igual que este estudio ${ }^{20}$. Para futuras investigaciones es indispensable conocer mejor la correlación entre la rata de sécreción salival, los factores que la alteran y la colonización por Candida. Se hace necesario tomar medidas preventivas enfocadas 
a la reducción de colonización de Candida en niños con leucemia que tengan una rata de secreción salival disminuída y así evitar complicaciones sistémicas. Con la realización de esta investigación sobre la composición de la microbiota subgingival existente y los cambios que presenta con la quimioterapia, es mucho más fácil proporcional un bienestar en la cavidad oral de pacientes con éste compromiso sistémico. No tenemos conocimiento de otras investigaciones en nuestro medio donde se estudie las condiciones periodontales y cómo se afectan con la quimioterapia en niños con L.L.A.

\section{CONCLUSIONES}

1. La mucositis y la gingivitis son las complicaciones orales más frecuentes en niños con leucemia linfocitica aguda entre los 6 y 12 años de edad que reciben quimioterapia en el Hospital Universitario del Valle.

2. El Fusobacterium spp. fue el microorganismo más prevalente en la microbiota subgingival de niños con L.L.A que reciben quimioterapia. Los cambios que induce la quimioterapia favorece una mayor colonización de este microorganismo teniendo una relación directa con la gingivitis.

3. Los bacilos entéricos gram negativos también fueron predominantes en la microbiota subgingival de estos pacientes, representando el $100 \%$ de la microbiota subgingival cultivada en seis de las muestras. Al existir una rata de secreción salival disminuida y concomitantemente una reducción del pH intraoral como consecuencia de las drogas antineoplásicas utilizadas, estos dos factores podrian favorecer una coninfección con este microorganismo.

4. La quimioterapia puede favorecer un ambiente subgingival propicio para el sobrecrecimiento de $P$. gingivalis y $P$. intermedia los cuales están involucrados en la enfermedad periodontal.

5. A medida que el flujo salival disminuye existe una tendencia a aumentar el recuento de levaduras en saliva lo cual podría generar una mayor susceptibilidad al desarrollo de candidiasis.

\section{SUMMARY}

The Acute Limphocytic Leukemia (ALL) can appear at any given age but is more common in children. The most frequent oral complications after initiating chemotherapy are mucositis, local infections, salival gland dysfunction, impairment of taste, pain and hemorrhages. The purpose of this investigation was to determine the effects of the chemotherapy on the subgingival microbiota and yeast count in saliva in children of 6-12 years old with A.L.L. in the Hospital Universitario del Valle. Twelve patients ( 8 male $/ 4$ female) who initiated their first cycle of chemotherapy and/or returned from a resting period of 3 months were included in the study. Subgingival microbial samples and non stimulated saliva samples were obtained from each patient at the beginning of the study and 30 and 60 days later. All information was analized using SSPS (ver 10.0) software for Windows and statistical analysis included Pearson test, Friedman test and Cochran test $(\mathrm{P}=0.05)$.

Mucositis and gingivitis were the most frequent oral complications observed during the 3 month period. Fusobacterium spp and gram negative enteric rods were found in high levels and frequency. A tendency of high yeast count was detected with diminished salivary secretion and this could partially explain a high susceptibility to Candida infections in these patients. Moreover, a decrease in salivary secretion and $\mathrm{pH}$ caused by chemotherapy may have influenced the overgrowth of Fusobacterium spp and gram negative enteric rods.

Keywords: Acute Limphocytic Leukemia, chemotherapy, subgingival microbiota, yeast count.

\section{BIBLIOGRAFIA}

1 Mariotti A. Dental Plaque-Induced gingival Diseases. Ann Periodontol 1999: 4; 7-19.

2 McKenna S.J. Leukemia. Oral Surg Oral Med Oral Pathol 2000; 89(2): 137- 141.

3 Sonis S, Fazio R, Fang L. Principles and practice of oral medicine. W.B. Saunders Company, $2^{\text {da }}$ edition, 1996, 426-454. 
4 Rolston KV, Bodey GP. Infections in patients with cancer. In: Holland JF, Frei E III, Bast RC Jr. Cancer Medicine. $3^{\text {nt }}$ ed. Philadelphia, Pa: Lea\&Febiger 1993: 2416-2441.

5 Pizzo PA, Meyers J, Freifeld AG et al. Infections in the cancer patient. In : DeVita VT, HeIlman S, Rosenberg SA, Eds: Cancer: Principles and Practice of Oncology. Phyladelphia: JB Lippincott Company, $4^{\text {th }}$ ed 1993:22922337.

6 Cannon RD, Holmes AR, Mason AB, Monk BC. Oral Candida: clearance, colonization or candidiasis? J Dent Res 1995;74:1152-1161.

7 Astor FC, Hanft KL, Ciocon JO. Xerostomia: a prevalent condition in the enderly. Ear Nose Throat J. 1999; 78: 476-479.

8 Screebny LM et al. Saliva: its role in health and disease. Int Dent J1992;42 suppl2: 291304.

9 Arendorf TM, Walter DM. The prevalence and intra-oral distribution of Candida Albicans in man. Arch Oral Biol 1980; 25: 1-10.

10 Odds FC. Candida and candidosis. $2^{\text {nd }}$ edn. London: Bailliere Tindall 1988: 104-107.

11 Papas AS, Russell D, Singh M, et al. Double blind clinical trial of a remineralizing dentifrice in the prevention of caries in a radiation therapy population. Gerondontol 1999;16:2-10.

12 Mandel ID. Sialochemistry in diseases and clinical situations affecting salivary glands. Crit Rev Clin Lab Sci 1980;12:321-366.

13 Löe $\mathrm{H}$. The gingival index, the plaque index systems. JPeriodontal 1963:26:610-616.

ENSAB III- III Estudio Nacional de Salud Bucal. II Estudio Nacional de Factores de Riesgo de Enfermedades Crónicas-ENFREC II: Tomo VII: Estudio Nacional de Salud Bucal. Colombia, 1999.

15 Hartroth B, Seyfarht I, Conrado G. Sampling of periodontal by paper points: evaluation of basic parameters. Oral Microbiol Immunol 1999;14(5):326-330.

16 Bretz W y col. Unstimulated salivary flow rates of young children. Oral Surg, Oral Med, Oral Patho 2001;91:541-545.

17. Torres RS et al. Relation between salivary flow rates and Candida counts in subjects with xerostomia. Oral Surg, Oral Med, Oral Patho 2002:93(2):149-154.

Epstein JB, Pearsall NN, Truelove EL. Quantitative relationship between Candida Albicans and the clinical status of human subjects. $J$ Clin Microbiol 1980;12:475-6.

19 Knox J, Puodziunas A, Feld R. Chemotherapy-induced oral mucositis; prevention and management. Drugs \& Aging 2002; 17:257-267.

20 Woo S-B, Sonis ST, Monopoli MM, Sonis AL.A longitudinal study of oral ulcerative mucositis in bone marrow transplant recipients. Cancer. 1993;72:1612-1617.

21 McCarthy GM, Awde JD, Ghandi Het al. Risk factors associated with mucositis in cancer patients receiving 5-fluorouracil. Oral Oncol 1998:34:484.

22 Dreizen S. Stomatotoxic manifestations of cancer chemotherapy. J Prosthet Dent 1978;40:650.

23 Saral R, Burns WH, Prentice HG. Herpes virus infections. Clinical manifestation and therapeutic strategies in immunocompromised patients. Clin Haematol 1984;13:645.

24 Wade JC, Day LM, Crowley JJ, Meyers JD. Recurrent infection with herpes simplex virus after marrow transplantation: role of specific immune response and acyclovir treatment. $J$ Infect Dis 1984;149:750.

25 Peterson DE. Pretreatment strategies in infection prevention in chemotherapy patients. Natl Cancer Inst Monogr 1990. 9:61.

26 Wahlin $\mathrm{YB}$, Hola AK. Changes in the oral microflora in patients with acute leucemia and related disorders during the periodo of induc- 
tion therapy. Oral Surg Oral Med Oral Pathol 1988;65:411-417.

27 Wahlin YB. Effects of chlorhexidine mouthrinse on oral health in patients with acute leucemia. Oral Surg Oral Med Oral Pathol 1989;68:279-287.

28 Main BE, Calman KC, Ferguson MM et al. The effect of cytotoxic therapy on saliva and oral flora. Oral Surg Oral Med, Oral Pathol 1984;58:545-548.

29 Ayars GH, Altman LC, Fretwell MD. Effect of decreased salivation and $\mathrm{pH}$ on adherence of Klebsiella species to human bucal epithelial cell. Infec Immun 1982;38:179-182.

${ }^{30}$ Moore WEC, Holdeman LV, Cato EP, Smibert RM, Burmeister JA, Palcanis KG, Ranney RR. Comparative microbiology of juvenile periodontitis. Infect Immun 1985;48:507519.

31 Moore WEC, Holdeman LV, Cato EP, Smibert RM, Burmeister JA, Palcanis KG, Ranney RR. Comparative microbiology of juvenile periodontitis. Infect Immun 1982; 48: 507 519.

32 Dahlén GG. Black-pigmented Gram negative anaerobes in periodontitis. FEMS .Immunol Med Microbiol 1993;6:181-192.

33 Contreras A, Doan N, Chen C, Rusitanonta T, Flynn MJ, Slots J. Importante of Dialister pneumosintes in human periodontitis. Oral Microbiol Immunol 2000;15(4):269-272.

34 Ashimoto A, Chen C, Bakler L, Slots J. Polymerase chain reaction detection of 8 putative periodontal pathogens in subgingival plaque of gingivitis and advances periodontitis lesions. Oral Microbiol Immunol 1996;11:266-273.

35 Socransky S, Haffafee A, Cugini M. Microbial complexes in subgingival plaque. J Clin Periodontol 1998;25(2) 134-144.

36 Moore WEC et al. Bacteriology of experimental gingivitis in children. Infect Immun 1985:46:1-6.
37 Goldenstein EJC, Tarenzi LA, Agyare EO, Berger JR. Prevalence of EIkenella corrodens in dental plaque. $J$ Microbiol 1983;17:636.639.

38 Ashimoto A, Chen C, Bakker I, Slots J. Polymerasa chain and advanced periodontitis lesions. Oral Microbiol Immunol 1996;11:266273.

39 Wahlin BY. Salivary secretion rate, yeast cells, and oral candidiasis in patients with acute leucemia. Oral Surg, Oral Med, Oral Patho 1991;71:689-695.

40 Silverman S Jr. Oral Cancer: complications of therapy. Oral Surg Oral Med Oral Pathol 1999;88:284-288.

41 Lockhart PB, Clark JR. Oral complications following neoadjuvant chemotherapy in patients with head and neck cancer. NCIMonogr 1990;9:99-101.

42 Wolf A,Moreira JE,Marmary Y, Fox PC. Lack of acute effects of methotrexate on rat parotid salivary gland function. Arch oral Biol. 1989;34:109-15

43 Baum BJ, Bodner L,Fox PC, Izutsu KT,Pizzo $\mathrm{PA}$, Wright WE. Therapy - induced dysfunction of salivary glands: implications for oral health.Espc Care dent 1985;5:274-7.

44 Schum CA,Izutsu KT, Molbo DM, Truelove EL, Gallucci B. Changes in salivary buffer capacity in patients undergoing cancer chemotherapy .J Oral Med 1979;34: 76-80.

45 Rhodes VA, Watson PM, Jonson MH. Association of chemotherapy-related nausea and vomiting with pretreatment and posttreatment anxiety. Oncol Nurs Form 1986;13:41-47.

46 Akerstedt T, Gillberg M, Hjemdahl P, et al. Comparison of urinary and plasma catecholamine responses to mental stress. Acta Physiol Scand 1983;117:19-26.

47 Bates JF, Adams D. The influence of mental stress on the flor of saliva in man. Arch Oral Biol 1968:13:593-6. 
48. Ueta E, Tanida T, Yoneda K, Yamamoto T, Osaka T. Increase of Candida cell virulence by anticancer drugs and irradiation. Oral Microbiol and Immunol 2001;16(4):243-249.

49 Umazume M, Ueta E, Osaki T. Reduced inhibition of Candida albicans adhesion by saliva from patients receiving oral cancer therapy. J Clin Microbiol 1995: 33: 432-439

50 Navazesh W, Wood G, Brightman VJ. Relationship between salivary rates and Candida Albicans counts. Oral Surg, Oral Med, Oral Patho 1995; 80: 284-288. st Jorge AOC, Totti MAG, Almeida OP, Scully C. Oral candidiasis established in the sialoadenectomised rat. $J$ Oral Pathol Med $1993 ; 22: 54-6$

52 Totti MAG, Jorge AOC, Santos EB, Almeida $\mathrm{OP}$, Scully $\mathrm{C}$. Implantation of Candida albicans and other Candida species in the oral cavity of rats. J Oral Pathol Med 1996;25:308-10.

53 Jansma J, Vissink A, Bruma J. A survey of prevention and treatment regimens for oral sequelae resulting from head and neck radiotherapy used in Dutch Radiotherapy Institures. Ins $J$ Rad, Oncol, Biol, Physics 1992; 24(2):359367.

Grafico 1. Tasa de secreción salival comparada en los tres periodos observacionales.

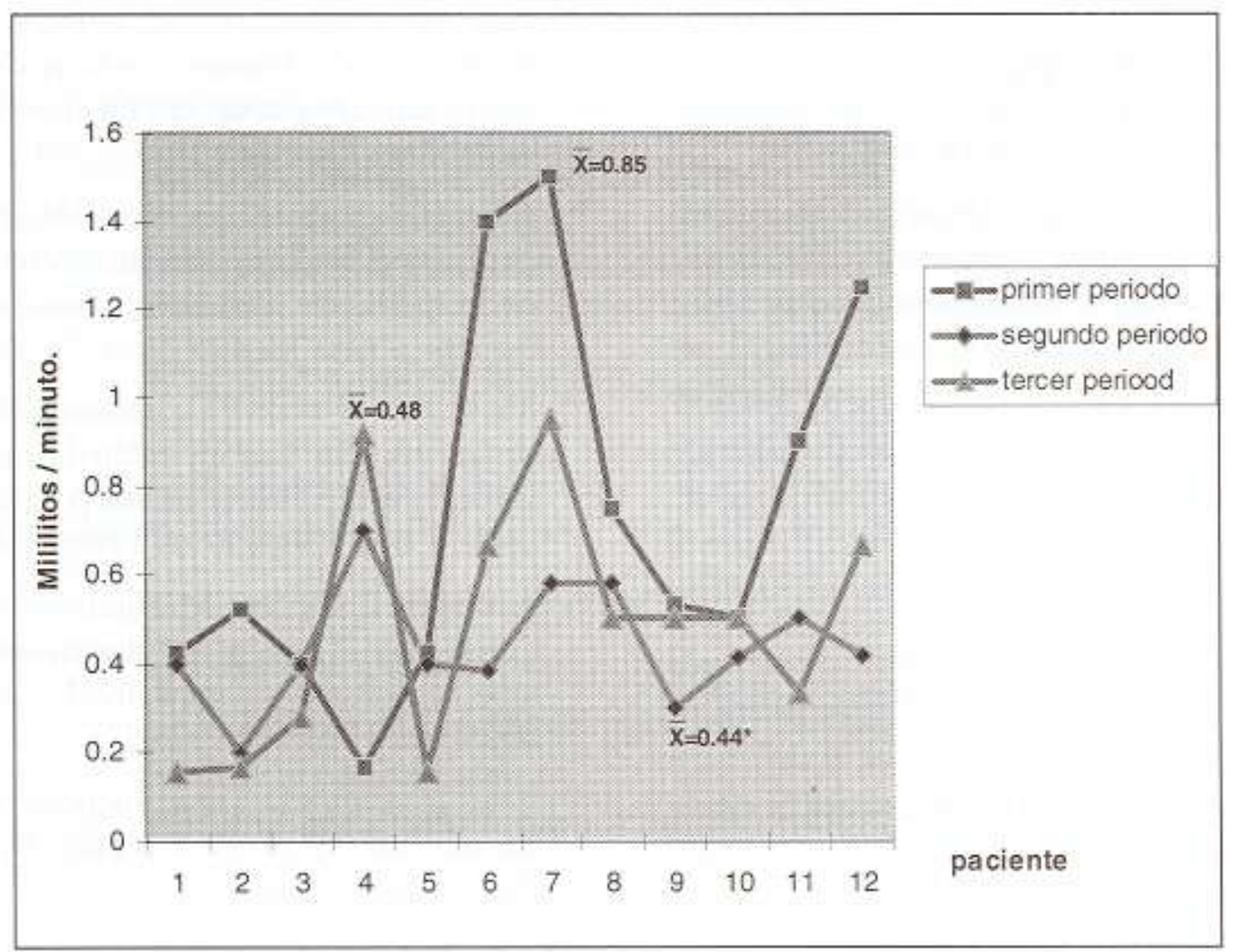


SEMANA

HDM

$2.0 \mathrm{gr} / \mathrm{m} 2 \mathrm{EV}$

ACIDO FOLINICO

$15 \mathrm{mg} / \mathrm{m} 2$ dosisi oral

TRIPLE INTRATECAL.

VTX 12 mgmz - ARA C $36 \mathrm{mg} / \mathrm{mz}$ dexametasona $5 m g / m 2$

VP 16

HD ARA C

$1 \mathrm{gr} 7 \mathrm{~m} 2 \mathrm{EV}$

VINCRISTINA

$1.5 \mathrm{mg} / \mathrm{m} 2 \mathrm{IM}$

L ASPARAGINASA

$10.000 \mathrm{U} / \mathrm{m} 2 \mathrm{EV}$

PREDNISOLONA

$40 \mathrm{mg} / \mathrm{m} 2$ oral

CICLOFOSFAMIDA

$800 \mathrm{mg} / \mathrm{m} 2 /$ dla SC:

ARABINOSIDO C

$50 \mathrm{mg} 7 \mathrm{~m} 27$ dia SC

METHOTREXATE

$15 \mathrm{mg} / \mathrm{m} 2$ orat

6 MERCAPTOPURINA $40 \mathrm{mg} / \mathrm{m} 27$ dia Oral

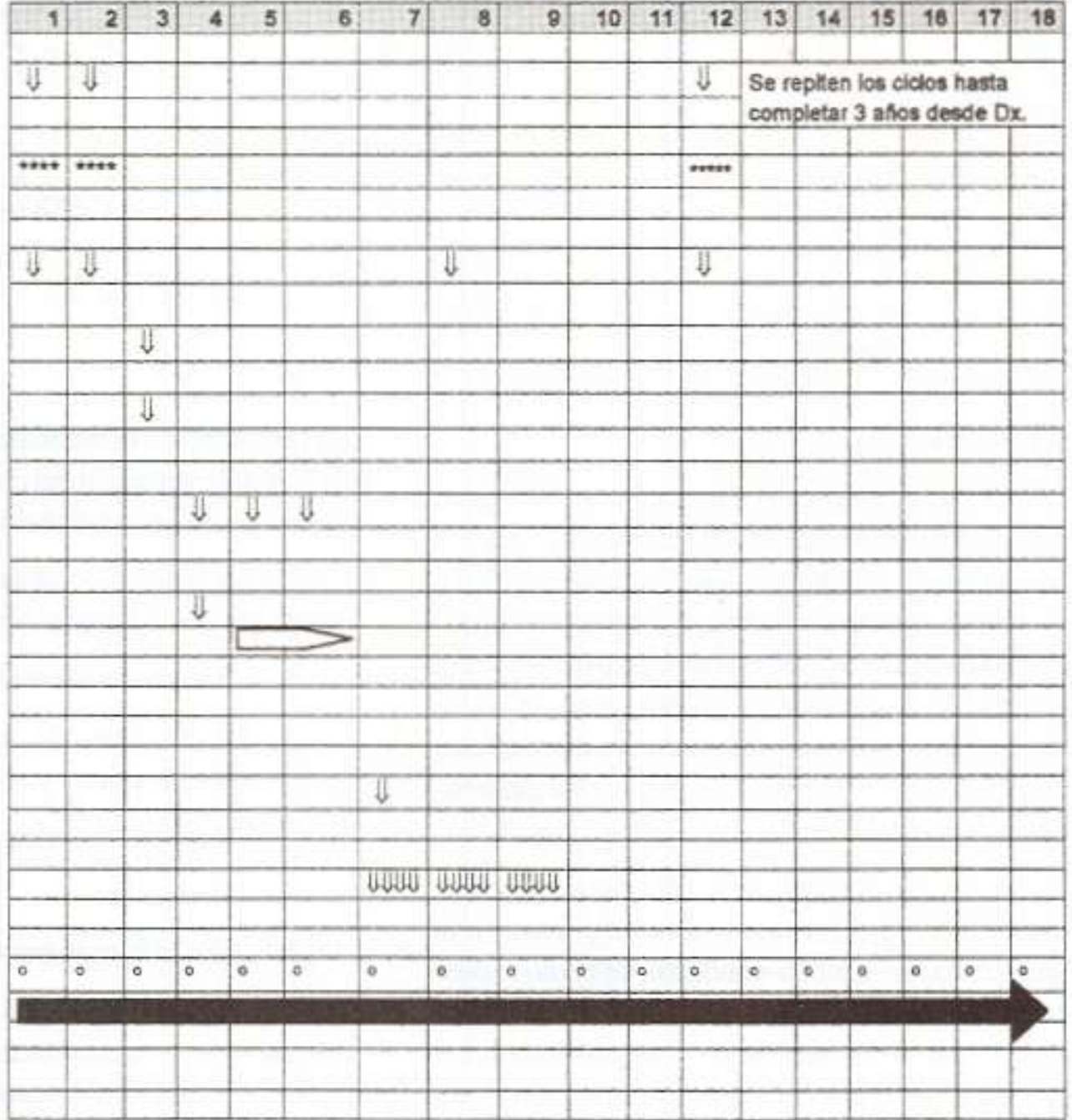

Correspondencia:

Adriana Jaramillo

Escuela de Odontologia, Universidad del Valle,

Cali, Colombia

Calle 4 B \# 36-00 San Femando

inv-odon@ univalle.edu.co 